\title{
Time-resolved full-field imaging of ultrasonic Lamb waves using deflectometry
}

\author{
C. Devivier*, F. Pierron, P. Glynne-Jones, M. Hill \\ Faculty of Engineering and the Environment, University of Southampton, Southampton SO17 1BJ, United Kingdom
}

\begin{abstract}
This pioneering experimental work is a proof of concept in which ultrasonic flexural waves have been imaged in a spatially and temporally resolved manner. Thin vibrating plates made of mirror glass and carbon/epoxy composite have been used in the experiments. Results obtained via a standard approach (scanning laser Doppler vibrometry) and the novel methodology based on deflectometry have been compared with a multi-physics finite element simulation. There is a very good correlation between the two experimental techniques. The numerical model provides insight into the experiments, but differs in its detailed structure due to uncertainties over material properties. The extreme slope resolution of deflectometry allows the measurement of peak-to-peak deflections of a few tens of nanometres in one shot. The use of an ultra-high speed camera allows for both space and time resolved measurements of Lamb waves which, to the best knowledge of the authors, has never been reported before. The limitations of the technique arise from the need for a flat specularly reflective surface. However, coating is possible for non-reflective materials and extension to moderately curved surfaces is possible in the future.
\end{abstract}

Keywords: piezoelectric transducer, ultrasonic Lamb waves, composites, ultra high-speed imaging, deflectometry

\section{Introduction}

This paper introduces a technique for imaging Lamblike flexural waves in a solid plate. Pure Lamb waves, able to propagate in free plates of infinite extent, were described by Horace Lamb in 1917 [1]. In these plate waves both free surfaces move simultaneously, in contrast to Rayleigh waves which penetrate a limited distance into the bulk from a single moving surface [2]. There are two types of Lamb waves as illustrated in figure 1 and of primary interest here is the antisymmetric, or flexural, wave.

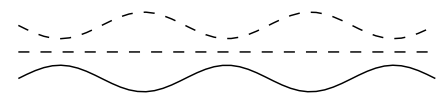

(a) Symmetric

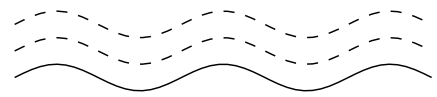

(b) Antisymmetric
Figure 1. Types of Lamb waves.

These waves have attracted a lot of attention as they can propagate easily over long distances which makes them sensitive to the presence of damage or defects. Ostachowicz et al. have recently published a review article on full wavefield signal processing for crack detection 3. In this article, the authors list three techniques that have been

\footnotetext{
* Corresponding author

Email addresses: cedric.devivier@gmail.com (C. Devivier), f.pierron@soton.ac.uk (F. Pierron), P.Glynne-Jones@soton.ac.uk (P. Glynne-Jones), M.Hill@soton.ac.uk (M. Hill)
}

used to measure the full wavefields: ultrasonic transducers, laser Doppler vibrometry (LDV) and shearography.

Ultrasonic transducers gather data at a single point where the transducer is attached and can be used for online structural health monitoring, for instance. An array of sensors can be used to triangulate the damage location by analysing the time signals. Each of these sensors provides temporally resolved information but many of them are required to obtain a spatially resolved information, and each provides local mass-loading to the structure. Therefore, this technology is not suitable to provide a full spatial description of the structural response. Scanning using one or more ultrasonic transducers moved over a surface is also possible [4, 5] but lacks the speed and flexibility of full-field approaches.

LDV also gathers information at a single point but uses the Doppler-effect: the frequency shift of the reflected laser light from a moving point on the surface measures its local velocity. It is therefore a non-contact method and can be automated to scan the plate over a predefined measurement grid. This is scanning laser Doppler vibrometry (SLDV) and it provides spatially-resolved information allowing a structure's operational deflection shape to be ascertained [6] as long as the excitation and specimen response are stable with time. Thanks to its relative ease of implementation, it has been extensively used, for example, in full wavefield signal processing [3] but also in damage detection using mechanical equilibria formulations at low 
frequency on beams 7, mid frequency 8 and high frequency 912 on plates. Scanning laser Doppler vibrometry provides similar data to the technique proposed here as long as the excitation and response are stable over a prolonged period of time. However, the scan time is generally long and the spatial resolution is typically limited to a few hundred data points for a reasonable scan time, which is not enough to capture local strain concentrations arising from the presence of defects, as seen for instance on delaminated plates [13, 14]. Finally, it is unsuited for capturing transient data.

Shearography is a full-field interferometric technique which measures surface slopes (spatial derivatives of deflection) [3. It has only been used to directly visualise damage such as cracks and delaminations or defects as these features reflect the incident waves. The reason behind this is the need for strobe lights which only allows the acquisition of data at a specific time to obtain the maximum magnitude, making this technique inconvenient for time-resolved measurements. Shearography provides spatially resolved information but temporal resolution is poor. Moreover, the equipment required for this technique is expensive and can be complex to use.

Among other full-field techniques, an obvious choice would be digital image correlation (DIC) [15]. It provides spatially and temporally resolved surface displacement maps by using cameras to track random markers attached onto the surface of the tested specimen. However, its displacement resolution is not sufficient for the current application. If one considers a correlation resolution of 0.01 pixels [16 and an sensor array of $1000 \times 1000$ pixels, one can therefore detect displacements of $0.01 / 1000=$ $1 \times 10^{-5}$ of the field of view. For a field of view of $60 \mathrm{~mm} \times$ $60 \mathrm{~mm}$ as is the case here, one has a resolution in out-ofplane displacements of $600 \mathrm{~nm}$, about 3 times larger than the maximum amplitudes measured in this work. To be able to image these waves, the resolution would need to be around $10 \mathrm{~nm}$ requiring a factor of 60 improvement. This may be achieved by spatial smoothing but assuming noise scales down with the square root of the width of the smoothing window, the smoothing window should be $60^{2}=3600$ data points wide which is more than three times larger than the number of independent displacement measurement points for a typical $30 \times 30$ pixel subset size.

The method adopted here is the grid method in deflectometry [13, 17 19. It provides spatially and temporally resolved surface slope maps by tracking the reflection of a regular grid pattern on the bent sample. This technique has an extremely high slope resolution. If one considers a resolution in slopes of $0.53 \mathrm{~mm} \mathrm{~km}^{-1}$ [14 and a spatial resolution of $1 \mathrm{~mm}$, one could theoretically have a resolution in out-of-plane deflections of $0.53 \times 10^{-6} \times 1 \times 10^{-3}=$ $0.53 \mathrm{~nm}$. Combined with ultra-high speed imaging, this technique is perfectly suited for this type of experiment.
DIC can also be used in specular reflection mode using a random pattern for the reflection target 20. This would improve the resolution of DIC, however, the spatial resolution is lower than that of grid-based deflectometry.

This paper presents a feasibility study on the spatially and temporally resolved visualization of ultrasonic flexural waves using a full-field slope measurement technique called deflectometry. To the best knowledge of the authors, it is the first time that this has been reported. It has the potential to provide a very valuable alternative to existing techniques to measure ultrasonic waves for NDT applications. The deflectometry measurements are used to generate operational deflection shapes for the plate which are compared to scanning laser Doppler vibrometry and numerical results. The first section describes the experimental set-up and data processing, then the finite element model is reviewed and the results are finally presented and discussed.

\section{Experimental set-up}

Two materials have been tested. The first one is a mirror made of glass with a reflective backing; the lateral dimensions are $152 \mathrm{~mm}$ by $110 \mathrm{~mm}$ and the thickness is $1.9 \mathrm{~mm}$. A piezoelectric transducer has been bonded onto the back of the mirror using an epoxy resin. The second material is a carbon/epoxy laminate, Hexcel IM78552. The lay-up is $[0 /+45 /-45 / 90]_{3 s}$ and the ply thickness is $0.125 \mathrm{~mm}$. The lateral dimensions of the carbon/epoxy plate are $200 \mathrm{~mm}$ by $140 \mathrm{~mm}$ and the thickness is $3 \mathrm{~mm}$. The piezoelectric transducer has been coupled onto the plate using a coupling gel and spring clamps. A gel coat has been applied onto the carbon specimen to obtain a smooth specularly reflective surface, according to the procedure detailed in [21]. This procedure was improved by adding an inert charge, carbon powder in this case, to the mix in order to ensure maximal opaqueness and avoid any parasitic reflections from the rough specimen surface under the coating [22].

The piezoelectric transducer is a square plate $(50 \mathrm{~mm}$ by $50 \mathrm{~mm}$ by $2.01 \mathrm{~mm}$ ) of Ferroperm PZ26 and is made of lead zirconate titanate (PZT). Figure 2 provides schematic of the samples (blue), the attached PZT (orange) and the regions of interest (ROI) imaged by the camera (green) and SLDV (red dashed line) for the glass panel only.

The experimental results have been obtained using the grid method in deflectometry. This method provides local surface slopes and is based on specular reflection of light as expressed in the Snell-Descartes law. Figure 3 presents a photo of the experimental set-up. The camera on the left-hand side is looking at the reflection on the sample of a printed grid pattern. The grid is a cross-hatch black and white pattern with a pitch of $2 \mathrm{~mm}$ and is shown as seen by the camera in the zoomed-in region in figure 3 . The grid 


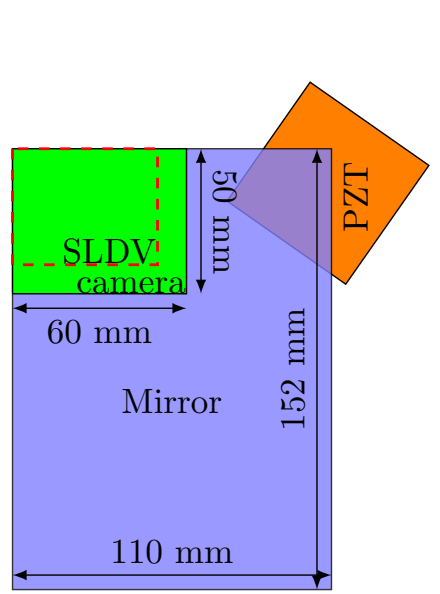

(a) Mirror glass

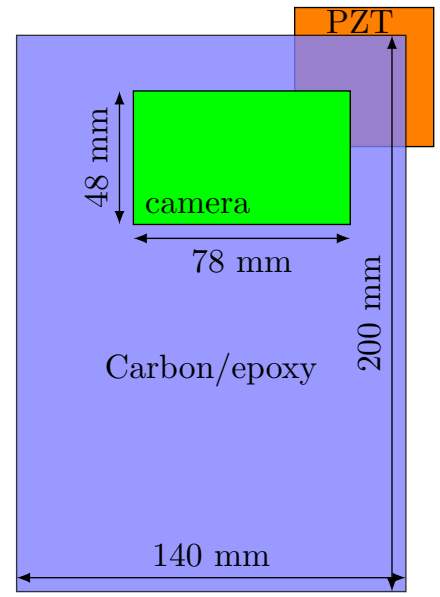

(b) Carbon/epoxy composite
Figure 2. Schematic of the samples with the piezoelectric transducer attached at the back and the various regions of interest, in green for the camera and in dashed red for the scanning laser Doppler vibrometer.

and the sample are around $1 \mathrm{~m}$ far away on the right-hand side. Two $500 \mathrm{~J}$ and two $200 \mathrm{~J}$ flash lights have been used to provide sufficient illumination during the tests. The PZT driver equipment, situated on the right-hand side, consisted of a sinusoidal signal generator, a power amplifier and an oscilloscope to check the excitation frequency and amplitude. The sample needed to be supported during excitation to make the loading more stable, but in a way that approximated free-free boundary conditions. This was experimentally achieved by covering two thirds across the width of the bottom $2 \mathrm{~cm}$ of the sample with a fluffy textile and lightly clamped as illustrated in the zoomed-in area.

Figure 4 presents the electrical impedance and phase spectra of the system "PZT bonded onto plate" for both mirror glass and carbon/epoxy composite. They were acquired by measuring the impedance at the PZT connections while sweeping through a frequency range using an impedance meter (C60 from Cypher Instruments). One frequency $(43.3 \mathrm{kHz})$ was selected for the mirror and two $(37.1 \mathrm{kHz}$ and $99.8 \mathrm{kHz})$ for the composite plate. They are highlighted as vertical dashed lines in figure 4. These frequencies are electrical impedance minima, and thus lie close to mechanical resonances of the system; by operating close to resonance, we achieve larger amplitude flexural waves. The lower frequencies were selected as they were expected to produce large deformation amplitudes and the much higher one to test the limits in terms of amplitude and frequency while maintaining the time resolution for the frame rate $(1 \mathrm{MHz})$.

A schematic of the principle of deflectometry is provided in figure 5. Since the specimen surface is specularly reflective, a given pixel of the camera images the reflection of point $P$. When the sample deforms in bending, there is a local slope change at point $M$ which causes a deflection of the ray of light. The same pixel now sees the reflection of point $Q$. The distance $M M^{\prime}$, denoted $h$, can be measured with a tape ruler and the distance $P Q$, denoted $d$, can be evaluated from the displacements of the grid lines. One can derive the following expression (see figure 5 for the definition of $\alpha$ and $\beta$ ):

$$
\begin{aligned}
\frac{d}{h} & =\frac{P Q}{M^{\prime} M}=\frac{P M^{\prime}+M^{\prime} Q}{M^{\prime} M}=-\frac{M^{\prime} P}{M^{\prime} M}+\frac{M^{\prime} Q}{M^{\prime} M} \\
& =-\tan (\beta)+\tan (\beta+2 \alpha) \\
& =-\tan \beta+\frac{\tan \beta+\tan (2 \alpha)}{1-\tan \beta \tan (2 \alpha)}
\end{aligned}
$$

Deflectometry is generally targeted at small deformations. When large deformations occur, the geometry changes and the data processing becomes much more complex. In the present case, the deformations caused by the flexural waves will be extremely small. Therefore, $\tan (\alpha)$ is much smaller than one and Eq. (1) can be simplified to:

$$
\frac{d}{h} \simeq-\tan \beta+\frac{\tan \beta+\tan (2 \alpha)}{1-\tan (2 \alpha)} \simeq \tan (2 \alpha)
$$

Since $\alpha$ is very small, $\tan (2 \alpha)$ is nearly equal to $2 \tan (\alpha)$. $\tan (\alpha)$ represents the local slope of the plate at point $\mathrm{M}$ with:

$$
\tan (\alpha) \simeq \frac{d}{2 h}
$$

In practice, $d$ will be measured through numerical processing of the grid images and therefore, $\tan (\alpha)$ will be provided by dividing $d$ by twice the grid to specimen distance. It must be pointed out that this expression is only valid when the distance $h$ is relatively large in comparison to the sample's dimension and the grid shifts. A new expression has been recently derived that does not suffer from such limitations [22] but it is unnecessary here. A nice feature of this measurement technique is that by increasing the grid to sample distance, one actually increases the slope resolution while keeping the same spatial resolution. There is however a physical limit to this increase which is dictated by the magnification and quality of the optical lens used on the camera.

Figure 6] shows the flow of the data processing for deflectometry to obtain strains and deflections from the grid images and hence to allow operational deflection shape analysis. A series of $n$ pictures are taken during the test. The first image is considered to be the reference. A further image is taken from the stack. The spatial phases along the horizontal and vertical directions of the reflected grid pattern are first extracted using a spatial phase shifting algorithm (windowed discrete Fourier transform) for both images [18, 23]. For both directions, the phase maps of the reference image are subtracted to that of the deformed images. They are then multiplied by $\frac{p}{2 \pi}$ to obtain 


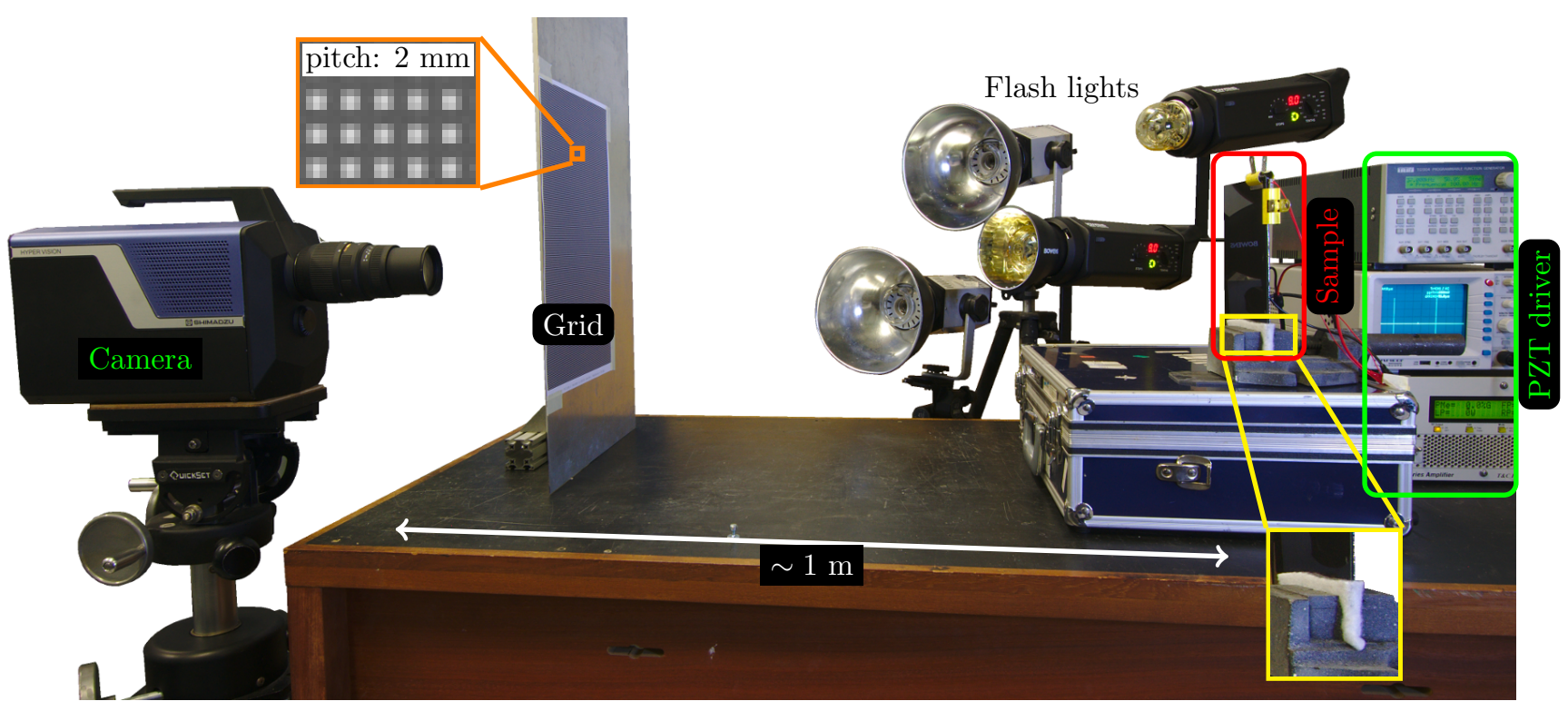

Figure 3. Experimental set-up and zoomed views of the grid and the simple support conditions.

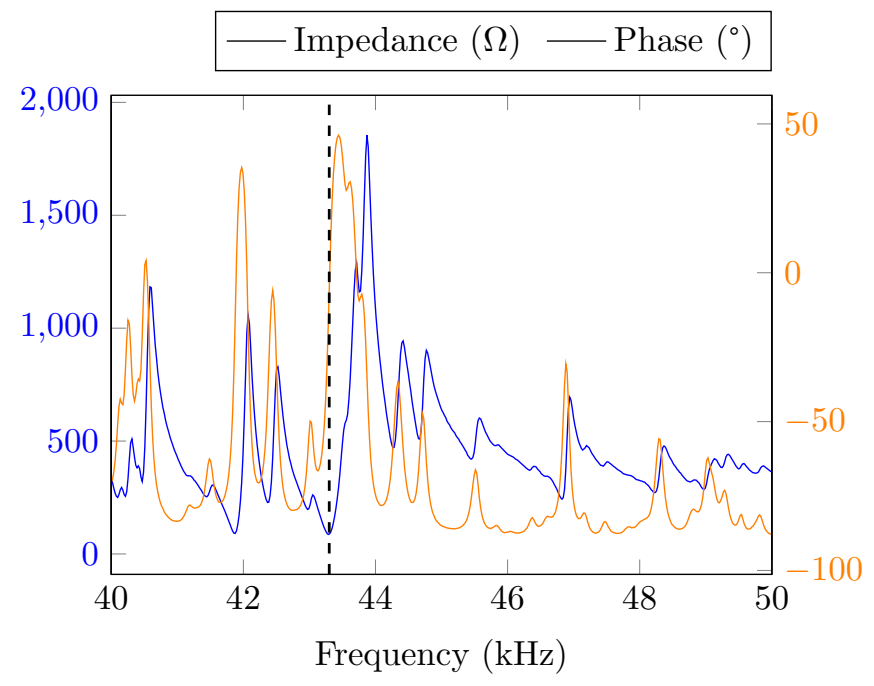

(a) Mirror glass

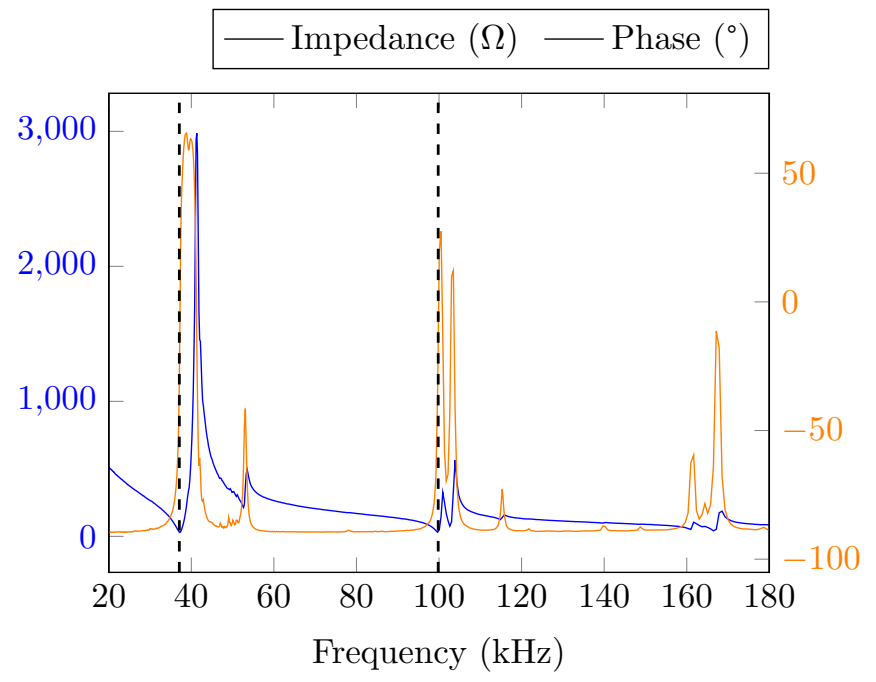

(b) Carbon/epoxy composite

Figure 4. Impedance and phase spectra of the PZT and plate system.

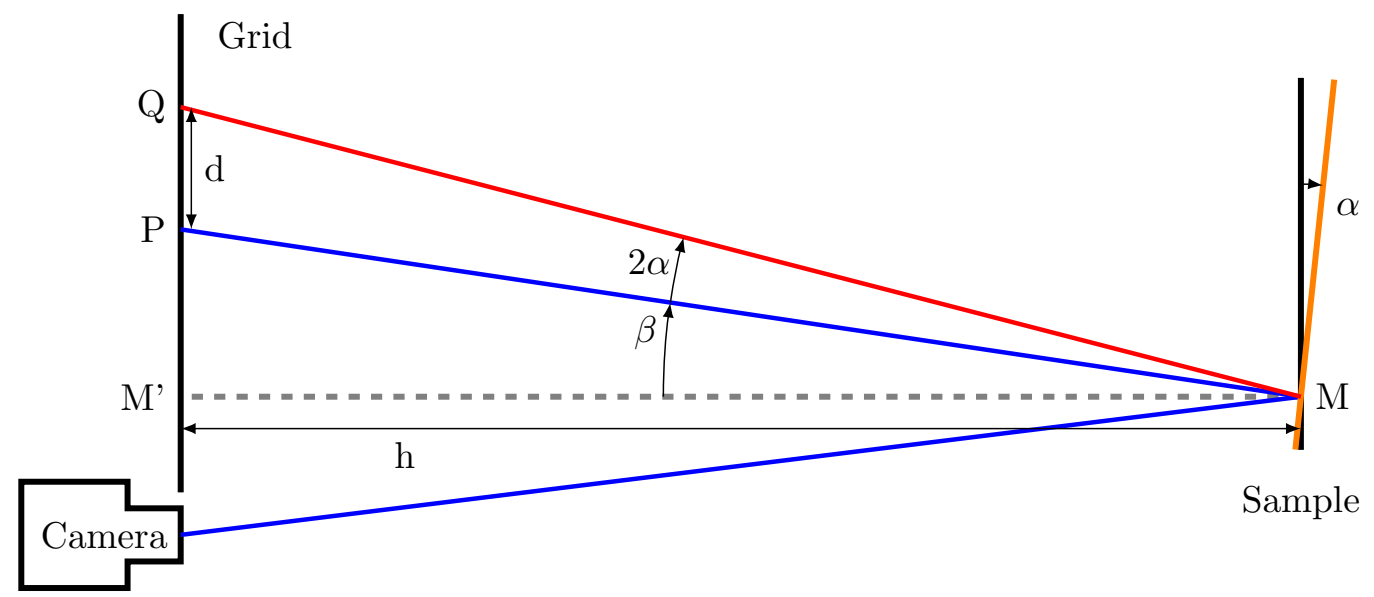

Figure 5. Principle of deflectometry 
the grid line displacement denoted $d$ with $p$ the pitch of the printed reference grid pattern. Using Eq. (3), the local slope changes along both directions are obtained. Curvatures are then computed by numerical differentiation using a centred finite difference algorithm. Equivalent strains are finally obtained by multiplying the curvatures by half the plate's thickness according to the Love-Kirchhoff thin plate theory. Strains are named "equivalent strains" as they rely on the thin plate assumptions. Wherever these assumptions hold, they are actual surface strains which will be the case here; but if they are violated they cannot be called strains any more, see [14. The deflection field is obtained by a least square numerical integration algorithm and setting the integration constant to zero as it corresponded to having the values on the nodal lines close to zero. This process is repeated for each images in the stack leading to $n-1$ sets of slope, strain and deflection maps.

In order to provide adequate temporal resolution at the required spatial resolution, ultra-high speed cameras have been used to capture grid images at rates between 0.5 and $1 \mathrm{MHz}$. Ultra-high speed imaging is defined in 24 in opposition to high-speed imaging where imaging rates are limited by memory read-out rates. The cameras used here rely on novel 'In-Situ Image Storage' sensors where the memory read-out limitation is alleviated by storing the electrons directly on the chip and reading the electron wells after the test. This enables the capture of about 100 images at rates up to $5 \mathrm{MHz}$ at an image size of about $100,000+$ pixels. These cameras have shown issues when used to perform deformation measurements, see for instance 25]. However, the new generation suffers much less from these problems and imaging quality has improved remarkably 26. These new technological developments make it possible now to record images at rates compatible with ultrasonic excitation, which significantly extends the coverage of techniques like deflectometry.

For the mirror sample, a Shimadzu HPV-2 ultra-high speed camera has been used to record a sequence of $102 \mathrm{im}-$ ages at $0.5 \mathrm{Mfps}$. It provides around 11.5 images per period over 9 periods with the $\mathrm{PZT}$ driven at $43.3 \mathrm{kHz}$. The first and last 12 images have been discarded because they are not reliable (well-known issue with this type of camera [25]) leaving 78 pictures to process. Its sensor array is 312 by 260 pixels. For the carbon/epoxy plate, a Shimadzu HPV-X ultra-high speed camera has been used to record a sequence of 128 images at $1 \mathrm{Mfps}$. This is a new generation FTCMOS sensor which suffers much less from the issues of the HPV-2 sensor, as shown in 26. It provides around 10 images per period over 13 periods with the PZT driven at $99.8 \mathrm{kHz}$ and 37 images per period over 5 periods with the PZT driven at $37.1 \mathrm{kHz}$. Its sensor array is 400 by 250 pixels. For both experiments, the grid pitch was $2 \mathrm{~mm}$ and the sampling was 5 pixels per grid pitch. The grid-sample distance is $0.85 \mathrm{~m}$ and $1.03 \mathrm{~m}$ for the mir- ror and the carbon/epoxy samples, respectively. Using these sets of images $(n=78$ or $n=128)$, the slope maps between image $i$ and the reference, image 1, have been computed. Additionally, the phase maps have been spatially smoothed just after the spatial phase shifting step using a Gaussian kernel of $3 \times 3$ and $2 \times 2$ pixels for the mirror and carbon/epoxy samples, respectively. It was necessary because the low fill factors of these cameras was causing aliasing problems in the horizontal direction, as reported in [25], even though the new Shimadzu HPV-X was less prone to this, hence the lighter smoothing. This also provided some extra noise reduction to improve the signal to noise ratio given the spectacularly low levels of deformation. Table 1 summarizes all the experimental parameters for both experiments and presents the noise floor values for the different physical quantities. The noise floor values were evaluated by first processing a series of grid images taken at rest using the same experimental conditions as a real test and following the work-flow described in figure 6. Then the spatial standard deviation of the phase, slope, strain and deflection maps were computed and the final values were obtained by temporally averaging these spatial standard deviations over the number of recorded images.

\begin{tabular}{lcc}
\hline Material & Mirror & Composite \\
\hline \hline Camera & HPV-2 & HPV-X \\
\hline Frame rate (Mfps) & 0.5 & 1 \\
\hline Number of pixels $\left(\mathrm{Pix}^{2}\right)$ & $312 \times 260$ & $400 \times 250$ \\
\hline \hline Grid pitch $(\mathrm{mm})$ & 2.032 & 2.032 \\
\hline Sampling $\left(\mathrm{Pix} \mathrm{period}{ }^{-1}\right)$ & 5 & 5 \\
\hline Grid distance $(\mathrm{m})$ & 0.85 & 1.03 \\
\hline Gaussian smoothing $\left(\mathrm{Pix}^{2}\right)$ & $3 \times 3$ & $2 \times 2$ \\
\hline \hline Noise floor phase $\left(\mathrm{mrad}^{2}\right)$ & 2.72 & 8.34 \\
\hline Noise floor slope $\left(\mathrm{mm} \mathrm{km}^{-1}\right)$ & 0.57 & 1.38 \\
\hline Noise floor strain $\left(\mu \mathrm{m} \mathrm{m}{ }^{-1}\right)$ & 0.25 & 1.41 \\
\hline Noise floor deflection $(\mathrm{nm})$ & 3.26 & 4.19 \\
\hline
\end{tabular}

Table 1. Experimental parameters for both experiments. The temporal fitting has not been included for the computation of the noise floors, therefore overestimating it.

The camera was triggered without any reference to the driving signal. This has the advantage of simplifying the experimental procedure by avoiding the need for synchronization of the camera to the excitation signal. However, the first grid image most likely corresponds to an already deformed state as a consequence. As the response is harmonic and of the same frequency as the excitation, as illustrated by figure 7, the complete series of slope maps can be used to identify the harmonic response. This was performed by fitting the following function at each data point of the slope maps using a least-square approach:

$$
S(x, y, t)=A(x, y) \sin (\omega t+\varphi(x, y))+B(x, y)
$$

with $A, B$, and $\varphi$, the amplitude, the offset, and the phase 


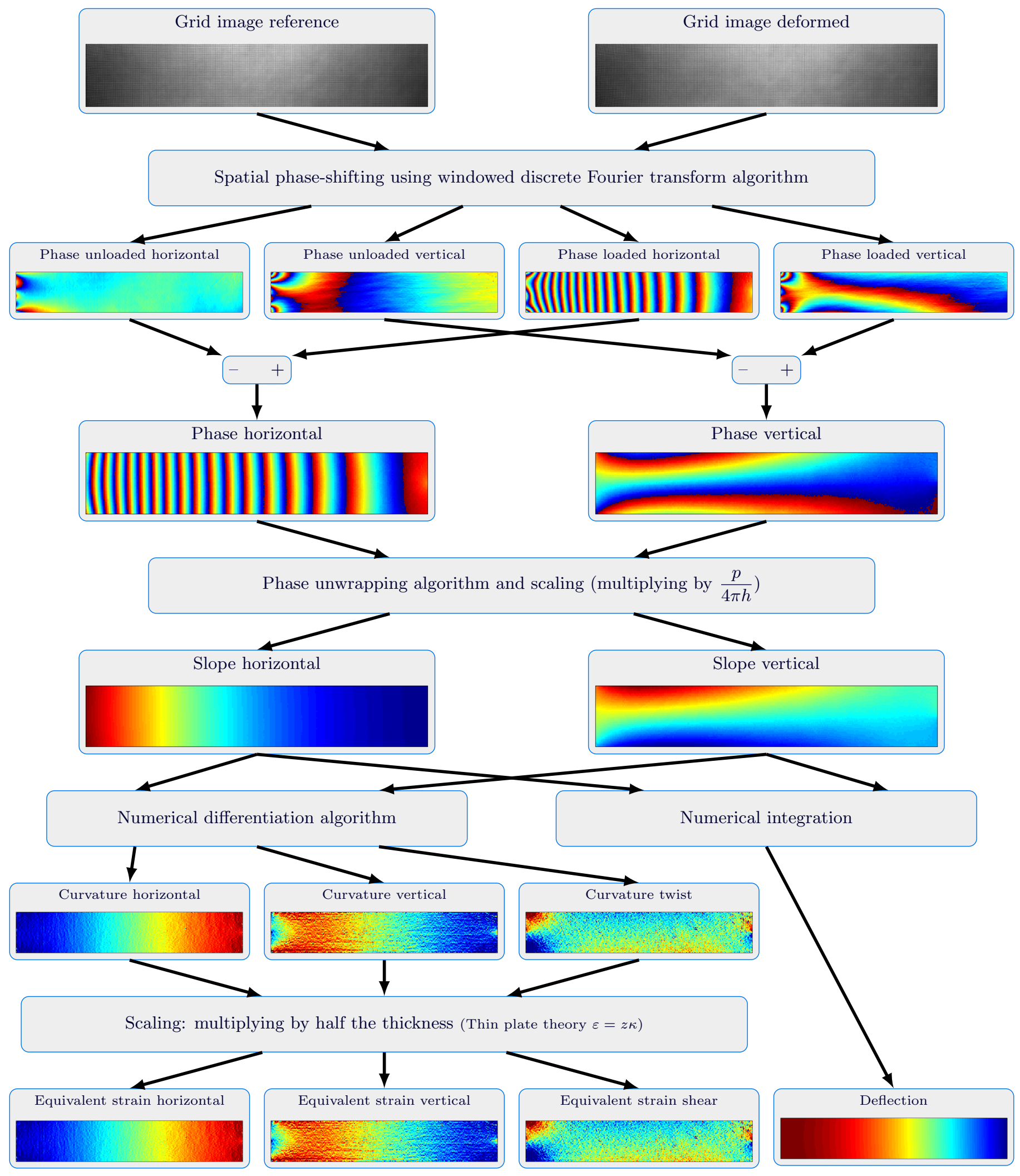

Figure 6. Data processing for deflectometry 
shift, respectively. Parameters $A, B$ and $\varphi$ have been identified at each data point so the complete response $S(x, y, t)$ could be reconstructed.

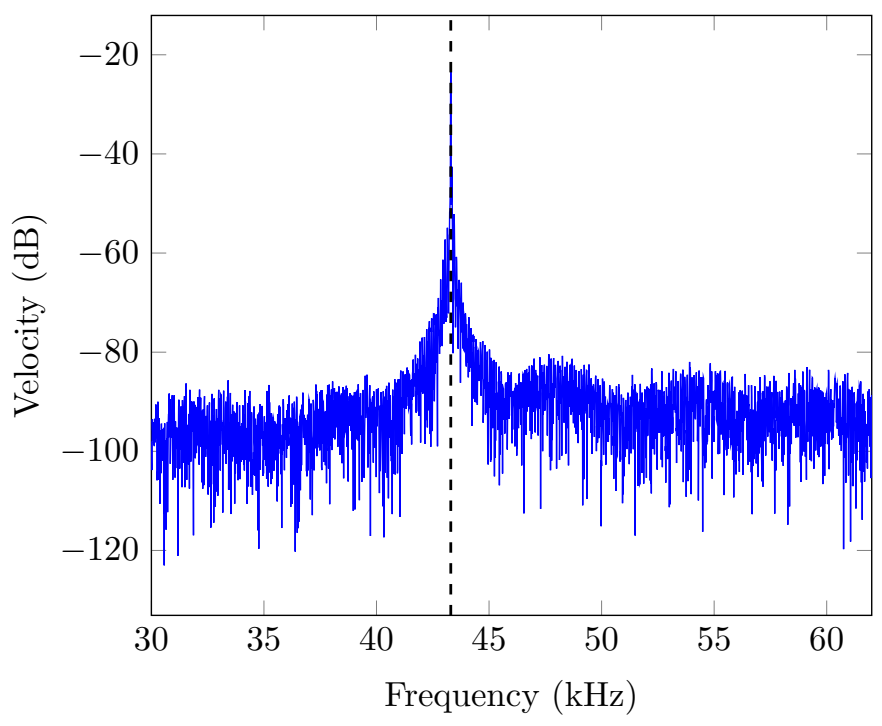

Figure 7. Velocity frequency response function of the excited glass mirror sample recorded by the vibrometer. Black dashed line indicates excitation frequency.

A scanning laser vibrometer (Polytec PSV-300) has also been used to validate the results with the glass mirror specimen. The scan of $15 \times 17$ data points took around 5 minutes to complete. The data have been resampled using a linear interpolation from $15 \times 17$ to $40 \times 50$ data points to be comparable with that from deflectometry $(50 \times 60$ data points). Because of this interpolation and the requirements of two numerical derivations, the strain fields are not significant. The field of view from deflectometry is slightly larger than that from the vibrometer, as shown in Figure 2.

\section{Finite element model}

The excitation of the plate by the piezoelectric transducer has been simulated using the ANSYS multiphysics finite element package. The PZT, the resin layer and the mirror plate have been modelled. The properties of the PZT have been taken from the manufacturer's dataset 27] and the driving voltage in the simulation matches the experimental values. The thickness of the resin layer was evaluated to be approximately $0.1 \mathrm{~mm}$. All of its properties have been taken as generic values for an epoxy resin. The properties of the mirror, such as Young's modulus, Poisson's ratio, and damping coefficient, have been chosen to be representative of an ordinary glass. The density has been obtained using the measured weight and dimensions of the mirror sample. Table 2 lists the properties of all materials used in the numerical simulations. It is expected that the numerical results will not match the experimental data because of the uncertainties in the material properties and the hypothesis that the mirror-resin and PZT-resin interfaces are perfect. It will however be useful to gain physical insight into the system.

The elements are all 20-node quadratic bricks with full integration. For the piezoelectric transducer, the element is fully-coupled, i.e. both piezoelectric and mechanical problems are solved simultaneously. The element reference number is SOLID226 with the first KEYOPT option set for electro-elastic. For the plate and the resin layer, the element is the classical mechanical one with the reference number SOLID186. The damping is introduced as proportional to the stiffness matrix divided by half the excitation angular frequency. The damping parameter is denoted $\eta$ in Table 2. It corresponds to the parameter $m_{j}$ in equation (14-22) from the theory reference of ANSYS 28. From the numerical results, the data have been retrieved in the same region as the deflectometry results. The sample was not constrained in displacements nor force. A zero voltage potential was applied on the surface of the PZT in contact with the bonding material (resin or coupling gel) and a harmonic potential with a magnitude of $130 \mathrm{~V}$ was applied on the other side of the PZT.

\section{Results}

This section presents the results for both samples.

\subsection{Mirror glass}

Figures 8 to 10 present the comparison between the SLDV, deflectometry, and numerical results. This comparison is performed on the operational deflection shapes $\left(U_{z}\right)$ figure 8 the horizontal and vertical slopes $\left(S_{x}\right.$ and $S_{y}$, respectively) figure 9 and the horizontal, vertical and shear equivalent strains $\left(\varepsilon_{x}, \varepsilon_{y}\right.$, and $\varepsilon_{s}$ respectively) figure 10. There is a very good correlation between the vibrometry (figures 8a, 9a and 9d) and deflectometry (figure $8 \mathrm{~b}$, 9b and $9 \mathrm{e} /$ results for the out-of-plane displacements and horizontal and vertical slopes. The operational deflection shapes are almost the same and the amplitudes match well, even though the SLDV slope data is lacking in spatial resolution and as a consequence, provide lower quality data than deflectometry. This is amplified when looking at the strain data obtained from the curvatures. The double spatial differentiation required for the SLDV data, together with the need for linear interpolation, result in poor strain information though both amplitudes and patterns show a very similar trend to the deflectometry data. The strains are spectacularly low, between 20 and -20 microstrains.

The finite element results exhibit a less striking correlation with the experimental results. In particular, the 


$\begin{array}{rlrlrlrl}\rho & 7750 \mathrm{~kg} \mathrm{~m}^{-3} & \varepsilon_{r}^{x} & 919 & \varepsilon_{r}^{y} & 919 & \varepsilon_{r}^{z} & 826 \\ \eta & 0.03 \% & C_{x x x x} & 120.0 \mathrm{GPa} & C_{y y x x} & 75.2 \mathrm{GPa} & C_{z z x x} & 75.1 \mathrm{GPa} \\ C_{y y y y} & 120.0 \mathrm{GPa} & C_{z z y y} & 75.1 \mathrm{GPa} & C_{z z z z} & 111.0 \mathrm{GPa} & C_{x y x y} & 22.6 \mathrm{GPa} \\ C_{y z y z} & 21.1 \mathrm{GPa} & C_{x z x z} & 21.1 \mathrm{GPa} & e_{x x z z} & -5.35 \mathrm{C} \mathrm{m}^{-2} & e_{y y z z} & -5.35 \mathrm{C} \mathrm{m} \mathrm{m}^{-2} \\ e_{z z z z} & +15.78 \mathrm{C} \mathrm{m}^{-2} & e_{y z y y} & +12.29 \mathrm{C} \mathrm{m}^{-2} & e_{x z x x} & +12.29 \mathrm{C} \mathrm{m}^{-2} & & \end{array}$

(a) Piezoelectric transducer 27

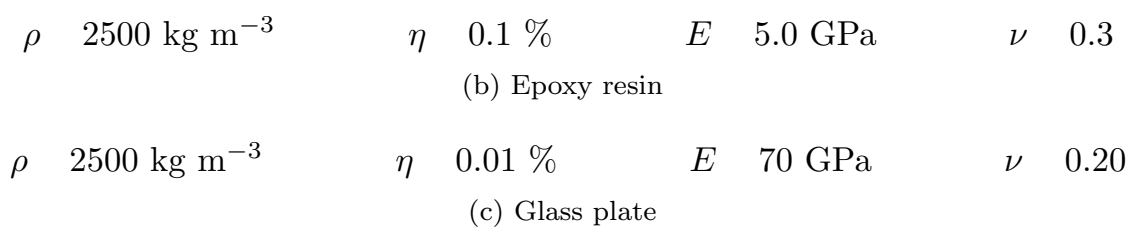

Table 2. Material properties used in the finite element model. $\rho$ is the density, $\varepsilon_{r}$ the directional relative permittivity, $\eta$ the structural damping ratio, $C_{i j k l}$ the stiffness coefficients, $e_{i j k k}$ the stress-charge coefficients, $E$ the Young's modulus, and $\nu$ the Poisson's ratio.

predicted amplitudes are one order of magnitude larger than the measured ones as expected. However, the spatial wavelengths are very similar, as well as the orientation of the wave patterns, which is reassuring. A sensitivity analysis has been performed to understand how much of these differences could be attributed to inadequate material properties. It will also help to understand the effect of each parameter on the global response of the specimen.

The results are presented in figure 11. The reference case is presented in figure 8b. The same colour scale has been kept to help visual comparison. Changing the plate's stiffness (figures $11 \mathrm{a}$ and $11 \mathrm{~d}$ ) modifies completely the wave patterns and the amplitudes. This is simply due to the fact that this modification changes the interaction between the shear and compressional waves that form these standing flexural wave patterns. The modification of the behaviour of the system due to the change in the plate's stiffness is clearly illustrated by the frequency response function presented in figure 12. Reducing the stiffness of the resin layer (figures $11 \mathrm{~b}$ and $11 \mathrm{e}$ induces slightly higher deflections but its thickness (figures 11c and 11f) did not change the behaviour greatly. It was expected that the resin layer played an important role in the results as it acts as a vibration damper because it is sandwiched between the excitation and the specimen. As expected, the damping coefficient (figures 11g and 11j) of the specimen has very little impact on the results. It was also checked that both the resin and the PZT damping had even less effects but the results are not presented here for conciseness. The mechanical (figures $11 \mathrm{~h}$ and $11 \mathrm{k}$ properties of the transducer have a critical effect on the system's behaviour whereas the piezoelectric coupling properties (figures 11i and 11l) have a much smaller influence. It should be noted here that the electro-mechanical coupling factor, also called electro-mechanical efficiency, has not been taken into account in the simulation. Its effect would be to reduce the global response. Figure 13 compares the impedance spectra of the modelled PZT to those measured experimentally close to the first thickness reso- nance. It can be seen that although the results are close, there is a small frequency error relating to an imperfect match in material parameters. We chose not to pursue closer matching of these properties, as uncertainties in the properties of the plate will also contribute to the variation between modelled and experimental results as shown previously. Moreover, the interface between the transducer and the plate being considered as perfect will also contribute to this mismatch. However, despite the differences observed the FE model is still valuable for understanding the type of wave propagation observed experimentally and the effect of the material properties. The qualitative agreement in spatial frequencies and wave patterns is enough to back-up the deflectometry measurements at this stage.

\subsection{Carbon/epoxy plate}

Figure 14 presents the deflectometry operational deflection shapes for excitations at $37.1 \mathrm{kHz}$ and $99.8 \mathrm{kHz}$. The data in the zone where the plate is connected to the piezoelectric transducer (top right corner) has been hidden. The values in this region were mainly driven by the PZT and were of much higher magnitude than that of the plate only. At $37.1 \mathrm{kHz}$, the carbon/epoxy plate exhibits a similar response to that of the glass plate at $43.3 \mathrm{kHz}$ shown in figure 8 . The spatial wavelengths are similar though the deformation amplitudes are lower, as expected from the fact that damping is higher in this polymer based material. Exciting the sample with a higher frequency causes the flexural wave patterns to change. The spatial wavelengths are now shorter and the amplitudes much smaller. These maps clearly illustrate the value of the deflectometry technique: providing very rich and detailed maps of extremely small deformations.

\section{Conclusions}

Deflectometry has been successfully used here to provide temporally and spatially resolved measurements of 


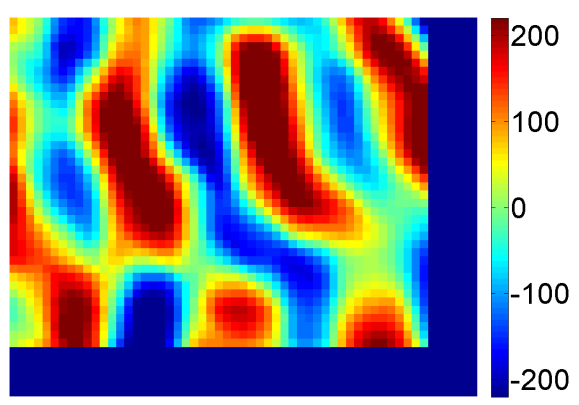

(a) $U_{z}(\mathrm{~nm})-\mathrm{SLDV}$

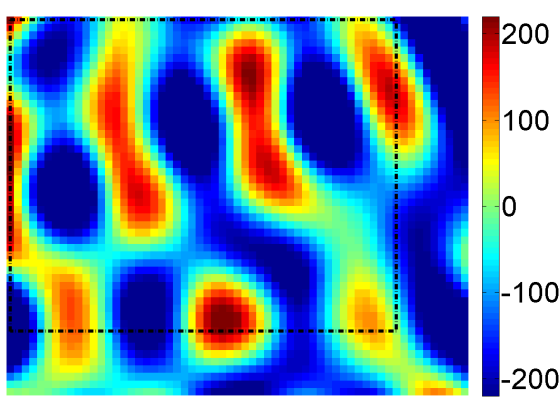

(b) $U_{z}(\mathrm{~nm})-$ Deflectometry

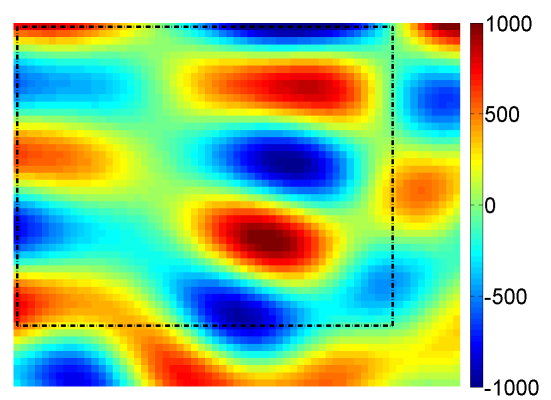

(c) $U_{z}(\mathrm{~nm})-\mathrm{FE}$ simulation

Figure 8. Comparison of the operational deflection shapes between scanning laser Doppler vibrometry, deflectometry and finite element simulation for the mirror sample excited at $43.3 \mathrm{kHz}$.

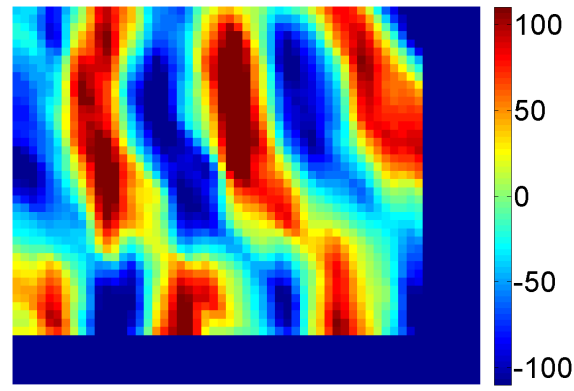

(a) $S_{x}\left(\mathrm{~mm} \mathrm{~km}^{-1}\right)-\mathrm{SLDV}$

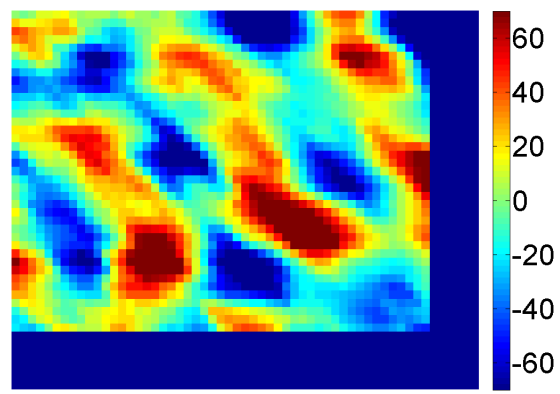

(d) $S_{y}\left(\mathrm{~mm} \mathrm{~km}^{-1}\right)-\mathrm{SLDV}$

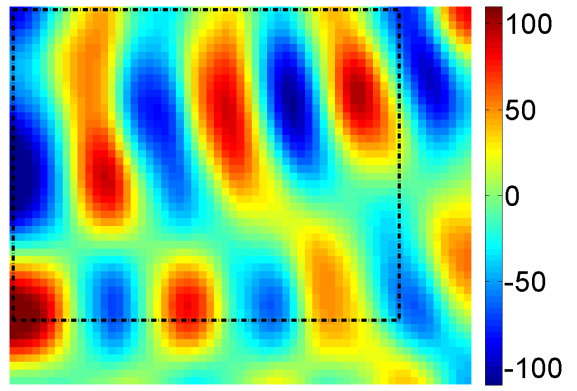

(b) $S_{x}\left(\mathrm{~mm} \mathrm{~km}^{-1}\right)-$ Deflectometry

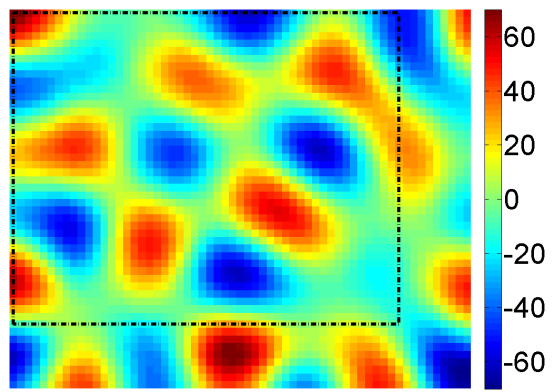

(e) $S_{y}\left(\mathrm{~mm} \mathrm{~km}^{-1}\right)-$ Deflectometry

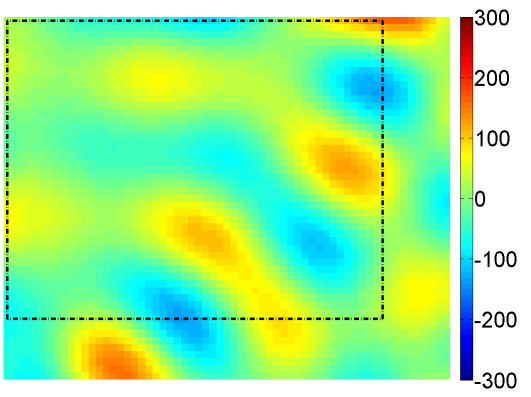

(c) $S_{x}\left(\mathrm{~mm} \mathrm{~km}^{-1}\right)-\mathrm{FE}$ simulation

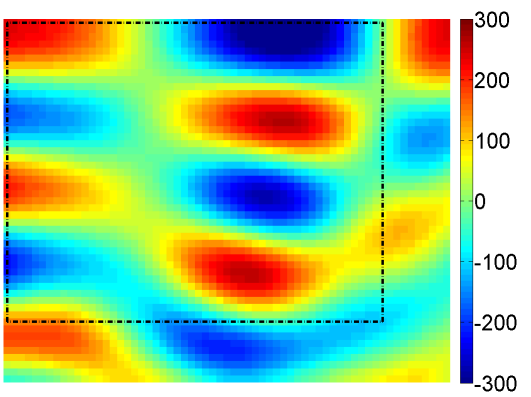

(f) $S_{y}\left(\mathrm{~mm} \mathrm{~km}^{-1}\right)-\mathrm{FE}$ simulation

Figure 9. Comparison of the horizontal and vertical slopes between scanning laser Doppler vibrometry, deflectometry and finite element simulation for the mirror sample excited at $43.3 \mathrm{kHz}$. 


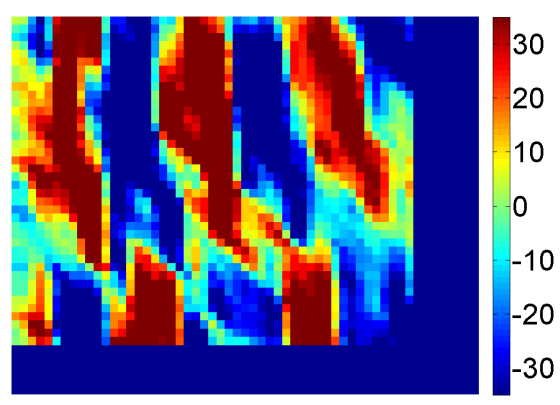

(a) $\varepsilon_{x}\left(\mu \mathrm{m} \mathrm{m}^{-1}\right)-\mathrm{SLDV}$

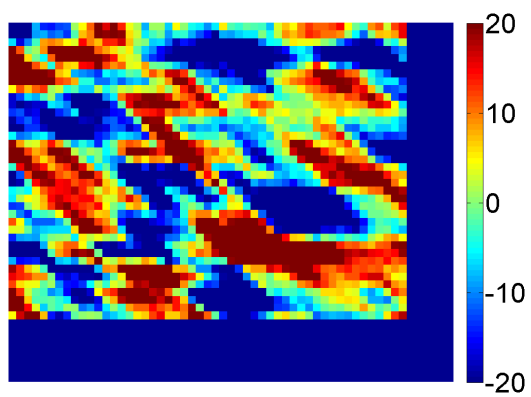

(d) $\varepsilon_{y}\left(\mu \mathrm{m} \mathrm{m}^{-1}\right)-\mathrm{SLDV}$

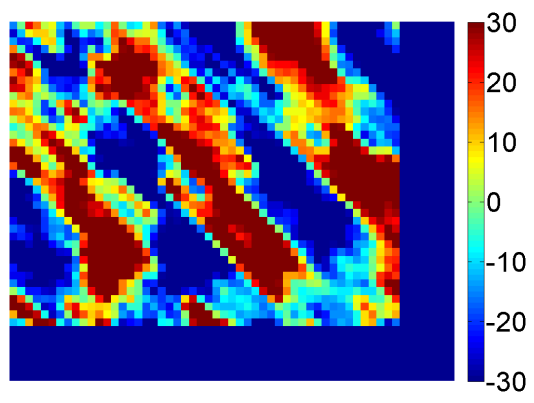

(g) $\varepsilon_{s}\left(\mu \mathrm{m} \mathrm{m}^{-1}\right)-\mathrm{SLDV}$

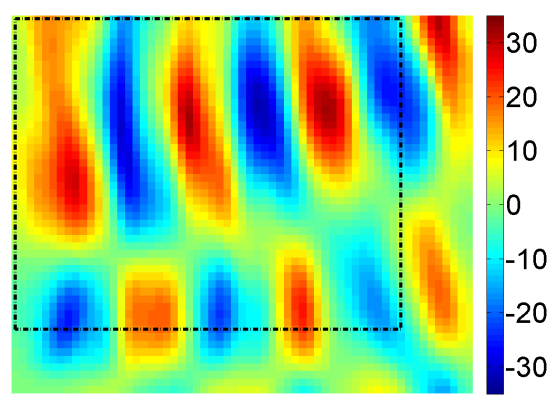

(b) $\varepsilon_{x}\left(\mu \mathrm{m} \mathrm{m}^{-1}\right)-$ Deflectometry

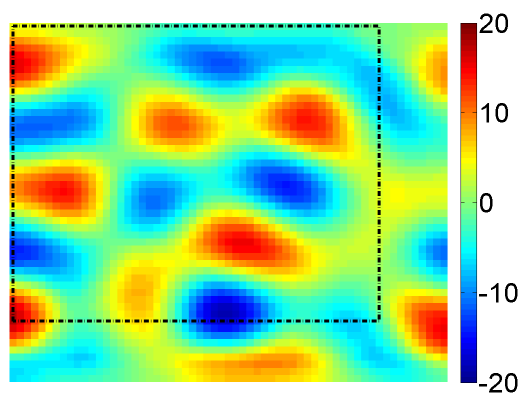

(e) $\varepsilon_{y}\left(\mu \mathrm{m} \mathrm{m}^{-1}\right)-$ Deflectometry

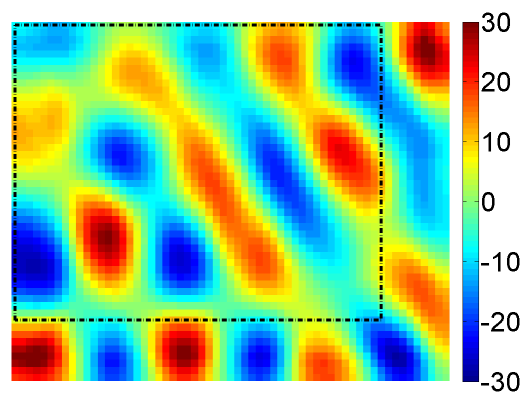

(h) $\varepsilon_{s}\left(\mu \mathrm{m} \mathrm{m}^{-1}\right)-$ Deflectometry

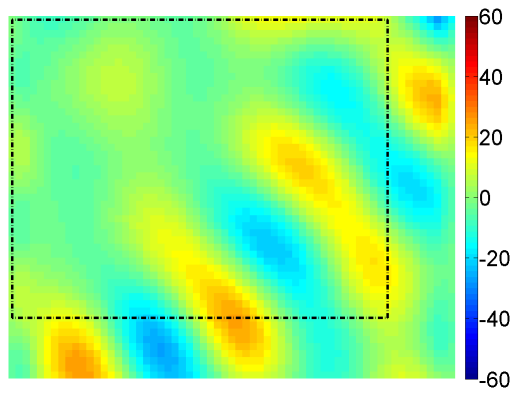

(c) $\varepsilon_{x}\left(\mu \mathrm{m} \mathrm{m}^{-1}\right)-\mathrm{FE}$ simulation

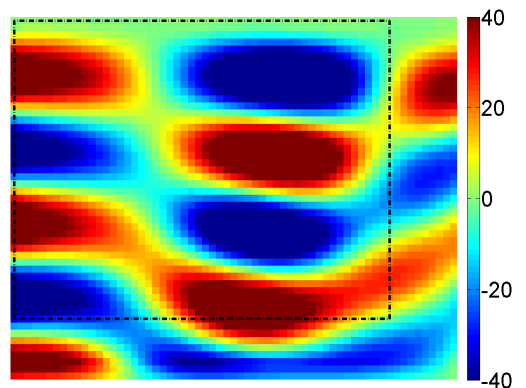

(f) $\varepsilon_{y}\left(\mu \mathrm{m} \mathrm{m}^{-1}\right)-\mathrm{FE}$ simulation

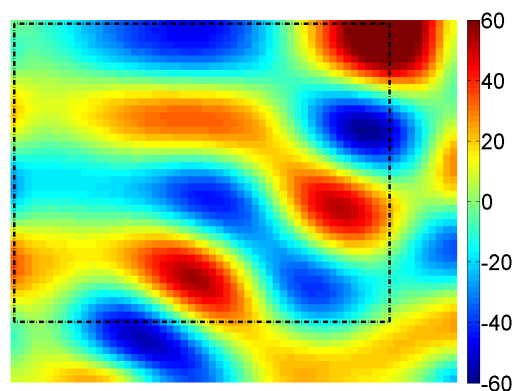

(i) $\varepsilon_{s}\left(\mu \mathrm{m} \mathrm{m}^{-1}\right)-\mathrm{FE}$ simulation

Figure 10. Comparison of the horizontal, vertical and shear equivalent strains between scanning laser Doppler vibrometry, deflectometry and finite element simulation for the mirror sample excited at $43.3 \mathrm{kHz}$. 


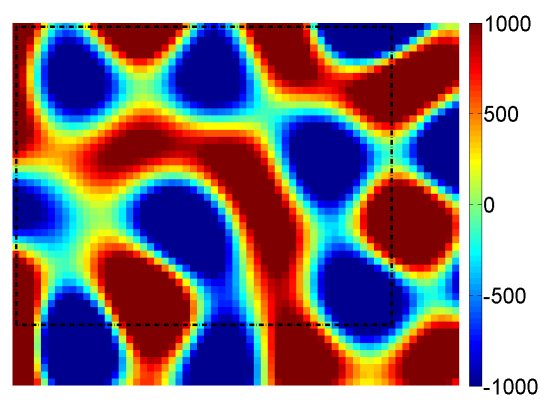

(a) $U_{z}(\mathrm{~nm})-\mathrm{RD}=+270.65 \%$ $E_{\text {pla }}=60 \mathrm{GPa}<E_{\text {pla }}^{\text {ref }}=70 \mathrm{GPa}$

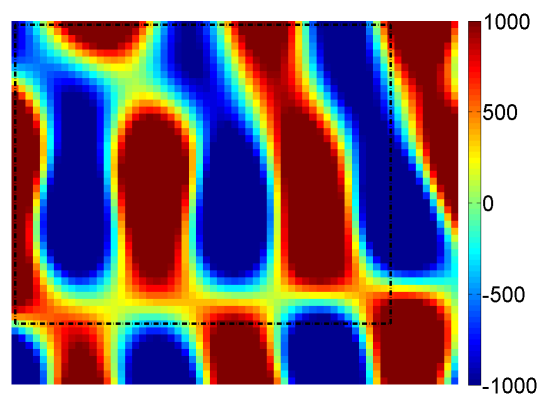

(d) $U_{z}(\mathrm{~nm})-\mathrm{RD}=+188.12 \%$

$E_{\text {pla }}=80 \mathrm{GPa}>E_{\text {pla }}^{\text {ref }}=70 \mathrm{GPa}$

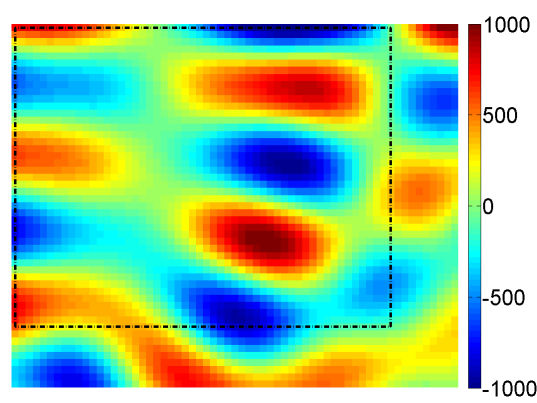

(g) $U_{z}(\mathrm{~nm})-\mathrm{RD}=+0.07 \%$

$\eta_{\text {pla }}=0.001 \%<\eta_{\text {pla }}^{\text {ref }}=0.01 \%$

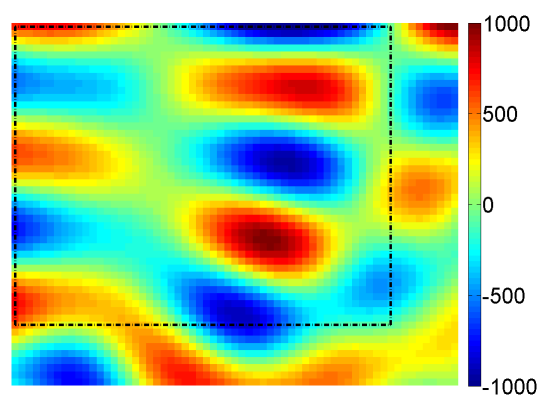

(j) $U_{z}(\mathrm{~nm})-\mathrm{RD}=-3.89 \%$

$\eta_{\text {pla }}=0.1 \%>\eta_{\text {pla }}^{\text {ref }}=0.01 \%$

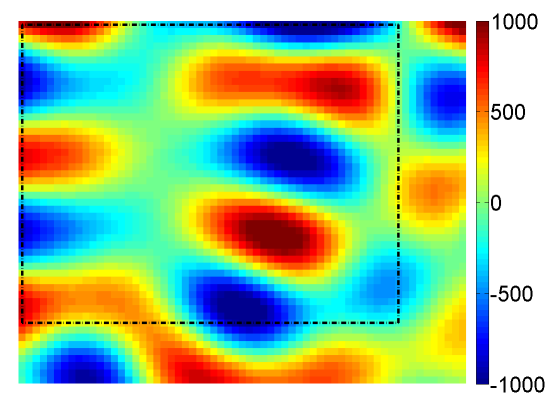

(b) $U_{z}(\mathrm{~nm})-\mathrm{RD}=+10.18 \%$

$E_{\mathrm{res}}=4 \mathrm{GPa}<E_{\mathrm{res}}^{\mathrm{ref}}=5 \mathrm{GPa}$

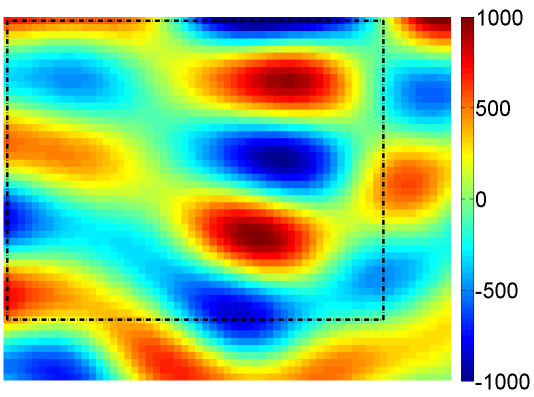

(e) $U_{z}(\mathrm{~nm})-\mathrm{RD}=+1.66 \%$

$E_{\text {res }}=6 \mathrm{GPa}>E_{\mathrm{res}}^{\text {ref }}=5 \mathrm{GPa}$

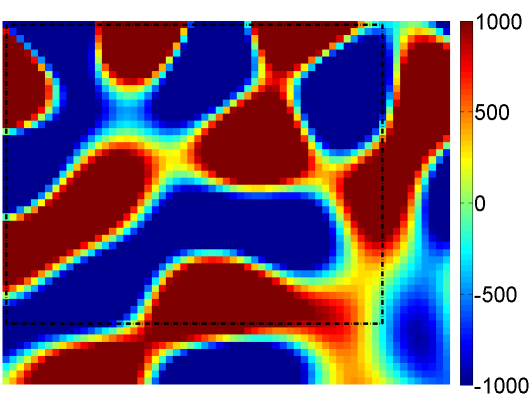

(h) $U_{z}(\mathrm{~nm})-\mathrm{RD}=+594.16 \%$

$E_{\mathrm{pzt}}=90 \%<E_{\mathrm{pzt}}^{\mathrm{ref}}=100 \%$

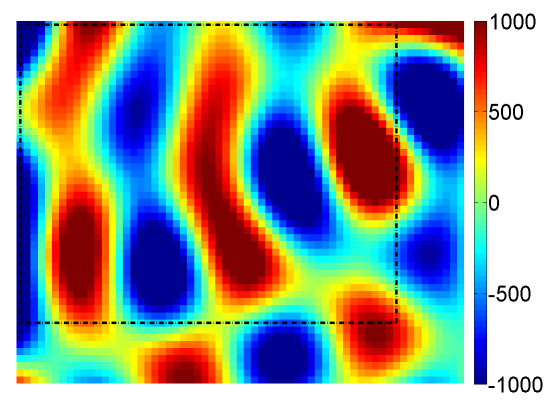

(k) $U_{z}(\mathrm{~nm})-\mathrm{RD}=+86.76 \%$

$E_{\mathrm{pzt}}=110 \%>E_{\mathrm{pzt}}^{\mathrm{ref}}=100 \%$

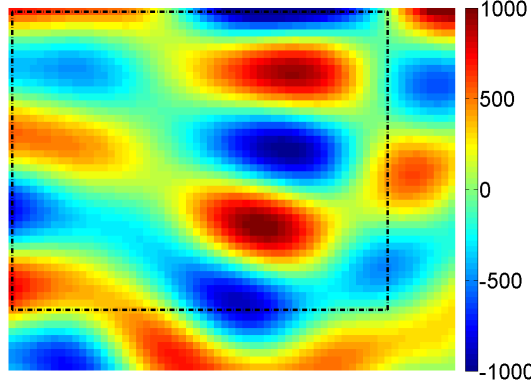

(c) $U_{z}(\mathrm{~nm})-\mathrm{RD}=+1.71 \%$ $t_{\mathrm{res}}=0.08 \mathrm{~mm}<t_{\mathrm{res}}^{\mathrm{ref}}=0.10 \mathrm{~mm}$

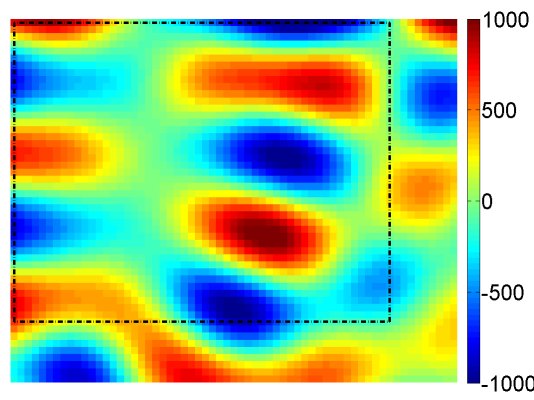

(f) $U_{z}(\mathrm{~nm})-\mathrm{RD}=+2.71 \%$

$t_{\mathrm{res}}=0.12 \mathrm{~mm}>t_{\mathrm{res}}^{\mathrm{ref}}=0.10 \mathrm{~mm}$

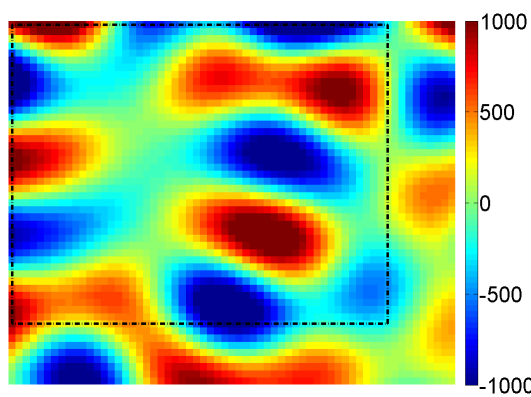

(i) $U_{z}(\mathrm{~nm})-\mathrm{RD}=+29.30 \%$

Elect $_{\mathrm{pzt}}=90 \%<$ Elect $_{\mathrm{pzt}}^{\mathrm{ref}}=100 \%$

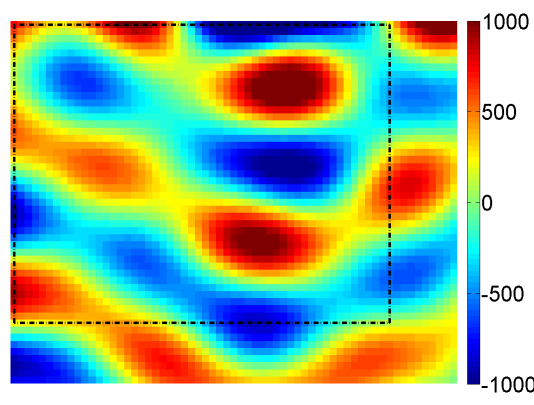

(l) $U_{z}(\mathrm{~nm})-\mathrm{RD}=+32.52 \%$

Elect $_{\mathrm{pzt}}=110 \%>$ Elect $_{\mathrm{pzt}}^{\mathrm{ref}}=100 \%$

Figure 11. Sensitivity analysis of the out-of-plane deflection in the operational deflection shapes to various parameters. RD is the relative difference of the absolute extrema between this case and the reference from figure $8 \mathrm{~b}$ A positive value indicates a higher value for the studied case and vice versa. 


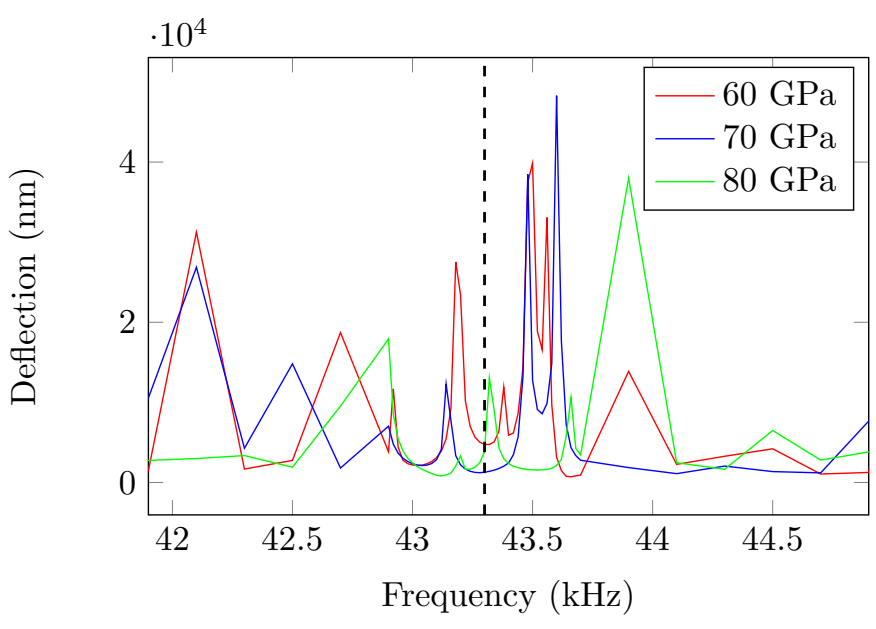

Figure 12. Frequency response function of the glass plate for different Young's moduli. The vertical dashed line indicates the excitation frequency.

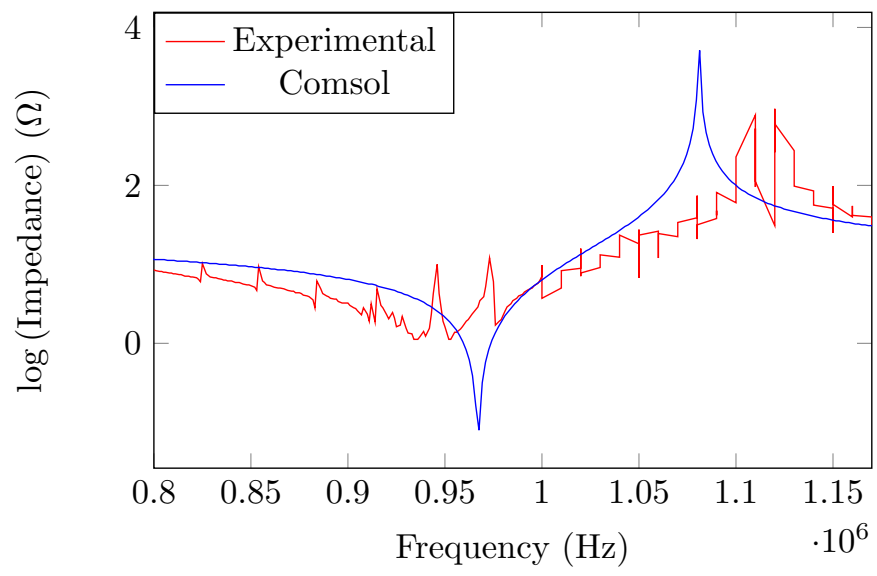

Figure 13. Frequency response function of the glass plate for different Young's moduli. The vertical dashed line indicates the excitation frequency. the operational deflection shapes caused by ultrasonic flexural waves propagating in mirror glass and carbon/epoxy composite samples. The results were corroborated by a standard measurement technique (scanning laser Doppler vibrometry, SLDV) in terms of amplitudes and spatial distributions, even though the quality of the deflectometry measurements proved far superior in terms of spatial resolution. The finite element simulation did not provide a full validation as the deformation amplitudes were an order of magnitude higher than the experimental ones, but the spatial wavelengths were consistent with the measurements. This is thought to be partly due to inaccuracies in the material parameters but probably more because of the difficulties in accurately representing the coupling between the PZT transducer and the panel. Since the objective here was not to develop a full finite element validation, this was not explored any further. It was also possible to investigate the effect of the excitation frequency on the response, up to $100 \mathrm{kHz}$.

To the best of the authors' knowledge, this is the first time that deflectometry has been used to image ultrasonic flexural waves. The key feature of deflectometry is that it measures slopes directly instead of deflections. This is extremely beneficial here as the curvatures (and hence, the strains in thin plate theory) are only one spatial differentiation away from the raw measurements, whereas the measurement of deflections requires a double spatial differentiation which strongly amplifies the effect of noise and compromises the curvature resolution. The main difference with existing techniques is that it provides temporal and spatial resolution simultaneously. The temporal resolution is limited by the frame rate of currently available ultra-high speed cameras, typically $0.2 \mu \mathrm{s}$ for the state-ofthe-art cameras. This enables one to resolve the waves at frequencies up to $100 \mathrm{kHz}$ at least. The spatial resolution of the measurements is limited by the spatial resolution of the camera sensor. The larger in this paper is $400 \times 250$ pixels $^{2}$ but another camera provides the same frame rate but with a $924 \times 768$ array sensor (Kirana camera, Specialized Imaging Ltd, UK). The spatial resolution also depends on the algorithm used to process the digital images. Here, the grid method enables to obtain an independent slope measurement point for each set of $5 \times 5$ pixels $^{2}$. Using a random speckle target and a digital image correlation matching, a larger window would be required, typically $20 \times 20$ pixels $^{2}$, leading to 16 times fewer data points. With the current setting using the Shimadzu HPV-X, the technique records the slopes simultaneously over a 50 by 80 grid. With SLDV, a grid of 15 by 17 measurement points was used, leading to a scan time of about five minutes. To provide the same spatial density as deflectometry, the scan time would have been about 80 minutes. Over such a long time, the stability of the specimen response becomes a key issue.

The main limitation of the present technique is that it 


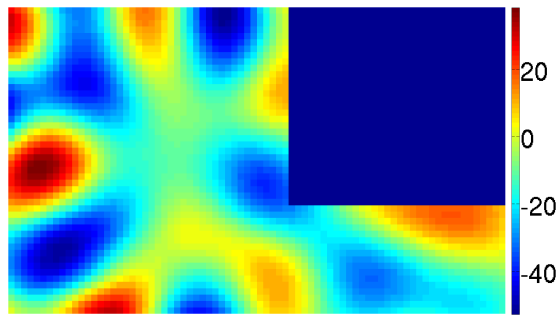

(a) $U_{z}(\mathrm{~nm})-37.1 \mathrm{kHz}$

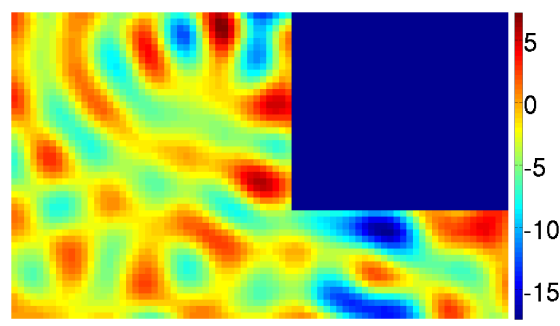

(d) $U_{z}(\mathrm{~nm})-99.8 \mathrm{kHz}$

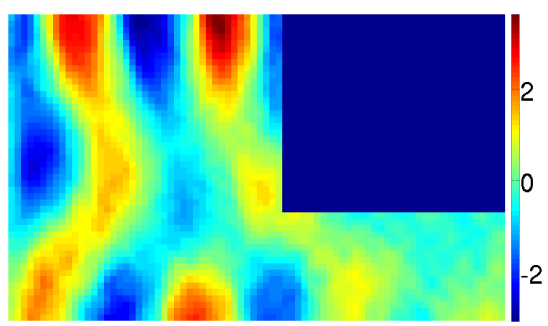

(g) $\varepsilon_{x}\left(\mu \mathrm{m} \mathrm{m}^{-1}\right)-37.1 \mathrm{kHz}$

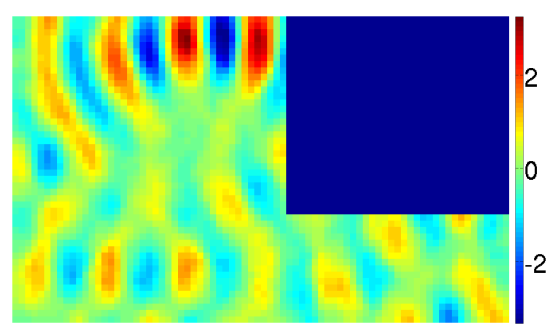

(j) $\varepsilon_{x}\left(\mu \mathrm{m} \mathrm{m}^{-1}\right)-99.8 \mathrm{kHz}$

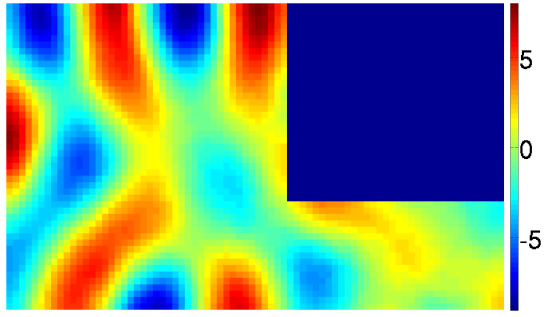

(b) $S_{x}\left(\mathrm{~mm} \mathrm{~km}^{-1}\right)-37.1 \mathrm{kHz}$

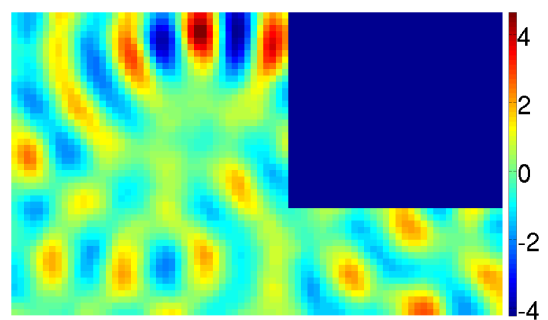

(e) $S_{x}\left(\mathrm{~mm} \mathrm{~km}^{-1}\right)-99.8 \mathrm{kHz}$

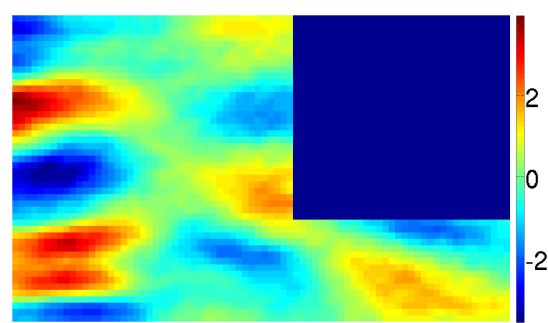

(h) $\varepsilon_{y}\left(\mu \mathrm{m} \mathrm{m}^{-1}\right)-37.1 \mathrm{kHz}$

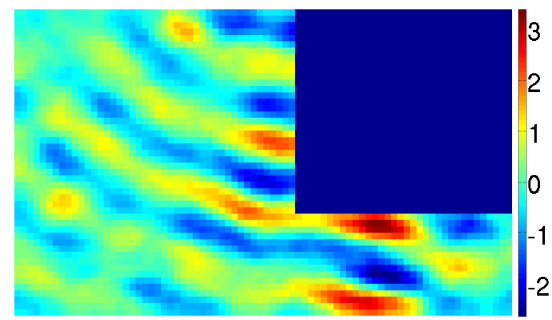

(k) $\varepsilon_{y}\left(\mu \mathrm{m} \mathrm{m}^{-1}\right)-99.8 \mathrm{kHz}$

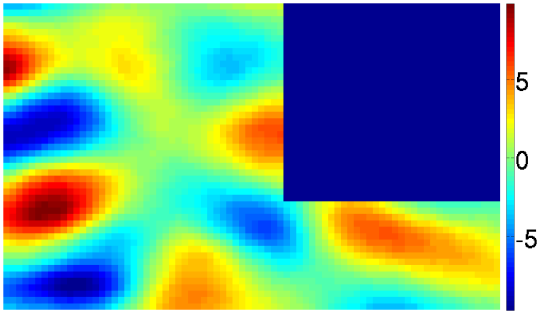

(c) $S_{y}\left(\mathrm{~mm} \mathrm{~km}^{-1}\right)-37.1 \mathrm{kHz}$

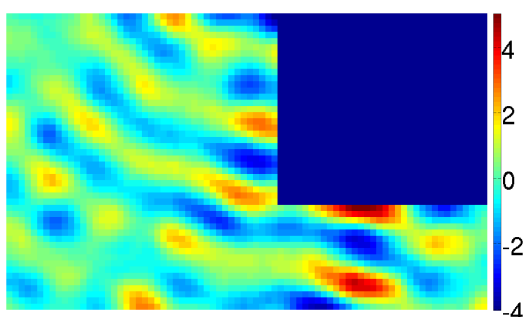

(f) $S_{y}\left(\mathrm{~mm} \mathrm{~km}^{-1}\right)-99.8 \mathrm{kHz}$

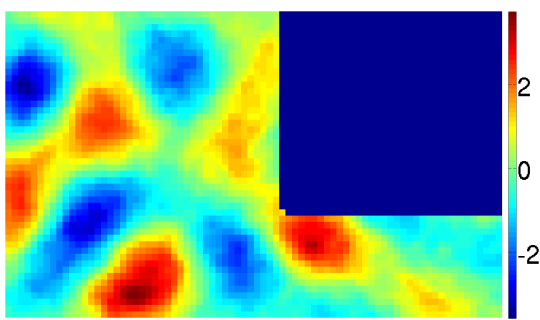

(i) $\varepsilon_{s}\left(\mu \mathrm{m} \mathrm{m}^{-1}\right)-37.1 \mathrm{kHz}$

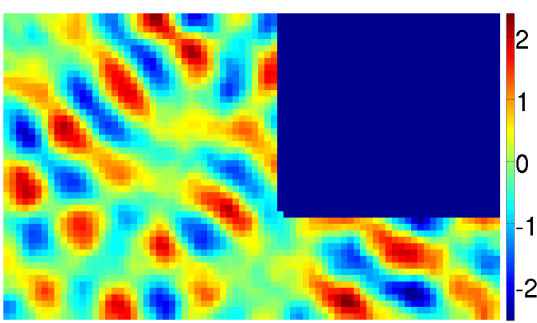

(l) $\varepsilon_{s}\left(\mu \mathrm{m} \mathrm{m}^{-1}\right)-99.8 \mathrm{kHz}$

Figure 14. Deflectometry operational deflection shapes for the carbon/epoxy sample at $37.1 \mathrm{kHz}$ and $99.8 \mathrm{kHz}$. $U_{z}$ is the out-of-plane displacement, $S_{x}$ and $S_{y}$ are the horizontal and vertical slopes, respectively and $\varepsilon_{x}, \varepsilon_{y}$, and $\varepsilon_{s}$ the horizontal, vertical and shear equivalent strains, respectively. 
is restricted to flat panels with smooth and highly specular reflective surfaces. The first requirement (flat surface) could be overcome by developing a calibration method suitable for this particular problem. This is difficult but not beyond reach as many studies have already been published on shape measurement of reflective surfaces, see 29] for instance. The second limitation is the need for a smooth and reflective surface. In the present work, the composite plate was coated with a thin layer of resin, according to the procedure developed in [21]. This would need to be extended to curved surfaces which should be possible, for instance using a smooth inflated silicone membrane which could be applied to the curved surface to mould the gel coat against. This means that the current technique has great potential for future NDT applications on structures like windmill blades or aerospace composite membranes, for instance. Finally, one of the challenges in NDT is to "read" these wave maps for the presence of damage. Recent damage indicators relying on mechanical equilibrium and dedicated to spatially resolved deformation data [14] could be adapted to this problem.

\section{Acknowledgements}

The authors would like to warmly thank the Engineering Instruments Pool 1 of the Engineering and Physics Sciences Research Council, Shimadzu ${ }^{2}$ and Speyer photonics ${ }^{3}$ for the loan of the cameras and flash lights. Professor Fabrice Pierron gratefully acknowledges support from the Royal Society and the Wolfson Foundation through a Royal Society Wolfson Research Merit Award. Doctor Peter Glynne-Jones gratefully acknowledges support from EPSRC under the Fellowship EP/L025035/1.

\section{References}

[1] H. Lamb, "On waves in an elastic plate," Proceedings of the Royal Society of London. Series A, vol. 93, no. 648, pp. 114128, 1917.

[2] Lord Rayleigh, "On waves propagated along the plane surface of an elastic solid," Proceedings of the London Mathematical Society, vol. s1-17, no. 1, pp. 4-11, 1885.

[3] W. Ostachowicz, M. Radzieǹski, and P. Kudela, "50th anniversary article: Comparison studies of full wavefield signal processing for crack detection," Strain, vol. 50, no. 4, pp. 275-291, 2014.

[4] P. Cawley, "Non-destructive testing - current capabilities and future directions," PROCEEDINGS OF THE INSTITUTION OF MECHANICAL ENGINEERS PART L-JOURNAL OF MATERIALS-DESIGN AND APPLICATIONS, vol. 215, no. L4, pp. 213-223, 2001.

[5] G. Dobie, A. Spencer, K. Burnham, S. G. Pierce, K. Worden, W. Galbraith, and G. Hayward, "Simulation of ultrasonic lamb wave generation, propagation and detection for a reconfigurable air coupled scanner," Ultrasonics, vol. 51, no. 3, pp. 258-269, 2011.

\footnotetext{
${ }^{1}$ http://www.eip.rl.ac.uk/

${ }^{2}$ http://www.ssi.shimadzu.com/

${ }^{3} \mathrm{http}: / /$ www.speyerphotonics.com/
}

[6] M. Martarelli and D. J. Ewins, "Continuous scanning laser doppler vibrometry and speckle noise occurrence," Mechanical Systems and Signal Processing, vol. 20, no. 8, pp. 2277-2289, 2006.

[7] H. Xu, L. Cheng, Z. Su, and J.-L. Guyader, "Identification of structural damage based on locally perturbed dynamic equilibrium with an application to beam component," Journal of Sound and Vibration, vol. 330, no. 24, pp. 5963-5981, 2011.

[8] H. Xu, L. Cheng, Z. Su, and J.-L. Guyader, "Damage visualization based on local dynamic perturbation: Theory and application to characterization of multi-damage in a plane structure," Journal of Sound and Vibration, vol. 332, no. 14, pp. 3438-3462, 2013.

[9] A. J. Romano, J. A. Bucaro, P. B. Abraham, and S. Dey, "Inversion methods for the detection and localization of inclusions in structures utilizing dynamic surface displacements," in Sixth International Conference on Vibration Measurements by Laser Techniques: Advances and Applications (E. P. Tomasini, ed.), SPIE, 2004.

[10] J. A. Bucaro, P. Abraham, A. J. Romano, and S. Dey, "Detection and localization of inclusions in plates using inversion of point actuated surface displacements," The Journal of the Acoustical Society of America, vol. 115, no. 1, pp. 201-206, 2004.

[11] J. A. Bucaro, J. F. Vignola, and A. J. Romano, "Fault detection and localization using laser-measured surface vibration," $N R L$ Review, pp. 81-88, 2005.

[12] J. A. Bucaro, A. J. Romano, J. F. Vignola, B. H. Houston, and P. B. Abraham, "Fault detection and localization using measured surface vibration and local inversion," in Nondestructive Evaluation and Health Monitoring of Aerospace Materials, Composites, and Civil Infrastructure V (A. A. Mufti, A. L. Gyekenyesi, and P. J. Shull, eds.), vol. 6176, SPIE, 2006.

[13] C. Devivier, F. Pierron, and M. R. Wisnom, "Damage detection in composite materials using deflectometry, a full-field slope measurement technique," Composites Part A: Applied Science and Manufacturing, vol. 43, no. 10, pp. 1650-1666, 2012.

[14] C. Devivier, F. Pierron, and M. R. Wisnom, "Impact damage detection in composite plates using deflectometry and the virtual fields method," Composites Part A: Applied Science and Manufacturing, vol. 48, no. 0, pp. 201-218, 2013.

[15] M. A. Sutton, J. J. Orteu, and H. W. Schreier, Image correlation for shape, motion and deformation measurements: basic concepts, theory and applications. Springer, 2009.

[16] G. Le Louëdec, F. Pierron, M. A. Sutton, and A. P. Reynolds, "Identification of the local elasto-plastic behavior of FSW welds using the virtual fields method," Experimental Mechanics, vol. 53, no. 5, pp. 849-859, 2013.

[17] Y. Surrel, N. Fournier, M. Grédiac, and P.-A. Paris, "Phasestepped deflectometry applied to shape measurement of bent plates," Experimental Mechanics, vol. 39, no. 1, pp. 66-70, 1999.

[18] Y. Surrel, "Deflectometry: a simple and efficient noninterferometric method for slope measurement," in Xth International Congress on Experimental \& Applied Mechanics, Society for experimental mechanics, 2004.

[19] A. Giraudeau, B. Guo, and F. Pierron, "Stiffness and damping identification from full field measurements on vibrating plates," Experimental Mechanics, vol. 46, pp. 777-787, 2006.

[20] C. Periasamy and H. V. Tippur, "A full-field reflection-mode digital gradient sensing method for measuring orthogonal slopes and curvatures of thin structures," Measurement Science and Technology, vol. 24, no. 2, p. 025202, 2013.

[21] J.-H. Kim, F. Pierron, M. Grédiac, and M. R. Wisnom, "A procedure for producing reflective coatings on plates to be used for full-field slope measurements by a deflectometry technique," Strain, vol. 43, no. 2, pp. 138-144, 2007.

[22] C. Devivier, Damage identification in layered composite plates using kinematic full-field measurements. PhD thesis, Université de Technologies de Troyes, 2012.

[23] Y. Surrel, "Fringe analysis," in Photomechanics (P. Rastogi, 
ed.), vol. 77 of Topics in Applied Physics, pp. 55-102, Springer Berlin / Heidelberg, 2000.

[24] P. L. Reu and T. J. Miller, "The application of high-speed digital image correlation," The Journal of Strain Analysis for Engineering Design, vol. 43, no. 8, pp. 673-688, 2008.

[25] M. Rossi, F. Pierron, and P. Forquin, "Assessment of the metrological performance of an in situ storage image sensor ultra-high speed camera for full-field deformation measurements," Measurement Science and Technology, vol. 25, no. 2, p. 025401, 2014.

[26] F. Pierron, H. Zhu, and C. Siviour, "Beyond hopkinson's bar," Philosophical Transactions of the Royal Society of London A: Mathematical, Physical and Engineering Sciences, vol. 372, no. 2023, 2014.

[27] "Ferroperm material properties dataset." http://app04.swwwing.net/files/files/Ferroperm\%20MatData.xls [Last accessed 13/01/2015].

[28] ANSYS 15.0.7 - Theory reference - 14.3.2 Harmonic (FULL) Analysis.

[29] J. Balzer and S. Werling, "Principles of shape from specular reflection," Measurement, vol. 43, no. 10, pp. 1305-1317, 2010. 